\title{
DKK4 enhances resistance to chemotherapeutics 5-Fu and YN968D1 in colorectal cancer cells
}

\author{
SHENGLI HE ${ }^{1}$, JIE SHEN ${ }^{1}$, NANHUA HU ${ }^{1}$, XUANFU XU ${ }^{2}$ and JIN LI ${ }^{3}$ \\ ${ }^{1}$ Oncology Department, Minhang Branch of Fudan University Shanghai Cancer Center, Shanghai 200240; \\ ${ }^{2}$ Gastroenterology Department, Tenth People's Hospital of Tongji University, Shanghai 200072; \\ ${ }^{3}$ Medical Oncology Department, Fudan University Shanghai Cancer Center, Shanghai 200032, P.R. China
}

Received July 13, 2015; Accepted September 6, 2016

DOI: $10.3892 / \mathrm{ol} .2016 .5461$

\begin{abstract}
Dickkopf-related protein 4 (DKK4) is a target of the $\beta$-catenin/transcription factor 4 complex in colorectal cancer. Previous research has demonstrated that its expression level may vary and has indicated that it may have a role in the development of resistance to chemotherapy in colorectal cancer cells. In the present study, DKK4 was over expressed in several colorectal cancer cell lines. The DKK4 over-expressing cell lines were screened using reverse transcription quantitative polymerase chain reaction analysis and western blotting. Analysis of cell viability in the control and DKK4 over-expressing cell lines, following treatment with 5-fluorouracil (5-Fu), YN968D1 or both, indicated that DKK4 over-expressing cells exhibit increased drug resistance. The results of Transwell chamber assays suggested that DKK4 had an effect on cell migration. Furthermore, the results from flow cytometric analysis showed that the percentage of apoptotic cells was reduced in the DKK4 over-expressing cell lines, following drug treatment, compared with the control. The present data suggested that DKK4 may enhance the resistance of colorectal cancer cells to 5-Fu and YN968D1 treatment, when used alone or in combination.
\end{abstract}

\section{Introduction}

Colorectal cancer is one of the most common types of cancer and is one of the leading causes of cancer-associated mortality worldwide (1). Numerous therapeutic approaches, including chemotherapy, radiotherapy and targeted therapy, have been used in the treatment of colorectal cancer (2). As one of the most common drugs used to treat colorectal cancer, 5-fluorouracil $(5-\mathrm{Fu})$ has the ability to induce apoptosis in normal

Correspondence to: Professor Jin Li, Medical Oncology Department, Fudan University Shanghai Cancer Center, 270 Dongan Road, Shanghai 200032, P.R. China

E-mail: 1ijin015@163.com

Key words: dickkopf-related protein 4,5-fluorouracil, YN968D1, chemotherapy resistance, colorectal cancer and tumor cells (3). However, clinical data has indicated that drug resistance to $5-\mathrm{Fu}$ is ubiquitous, even when $5-\mathrm{Fu}$ is used in combination with other chemotherapeutic agents $(4,5)$. YN968D1 is a tyrosine kinase inhibitor that selectively inhibits vascular endothelial growth factor (VEGF) receptor 2 (6). YN968D1 is thought to function by inhibiting VEGF-mediated endothelial cell migration and proliferation (7). As YN968D1 inhibits angiogenesis in cancer cells, it may be a novel treatment option for advanced types of cancer, including colorectal cancer.

Dickkopf-related protein 4 (DKK4) is a member of the dickkopf family of genes, which have been implicated in tumorigenesis. DKK4 is a target of the $\beta$-catenin/transcription factor 4 complex and binds to the Wnt co-receptors, low-density lipoprotein receptor-related protein 5 and 6 (8). DKK4 is overexpressed in the colonic mucosa of patients with colitis (9), while the expression of DKK4 is downregulated in colorectal cancer cells (10). Previous research has shown that DKK4 may be involved in resistance to chemotherapy in colorectal cancer (9). To further understand the role of DKK4 in chemotherapy resistance, the present study induced its overexpression in several colorectal cancer cell lines, which were subsequently analyzed for cell viability, migration and apoptosis following treatment with 5-Fu, YN968D1 or both. The results of the present study suggest that DKK4 may enhance the resistance of colorectal cancer cells to 5-Fu and YN968D1 treatment, when used alone or in combination.

\section{Materials and methods}

Cell culture and treatment. A total of 6 human colorectal cell lines were used: HCT116, HT29, LoVo, SW480, Caco2 and Colo205. All cell lines were purchased from the Cell Bank of Type Culture Collection of Chinese Academy of Sciences, Shanghai Institute of Cell Biology (Shanghai, China). All cells were cultured in Dulbecco's modified Eagle's medium (Gibco; Thermo Fisher Scientific, Inc., Waltham, MA, USA) supplemented with $10 \%$ calf serum (PAA Laboratories; GE Healthcare Life Sciences, Chalfont, UK) and treated with 5-Fu (Sigma-Aldrich; Merck Millipore, Darmstadt, Germany), YN968D1 (Jiangsu Hengrui Medicine Co., Ltd., Lianyungang, China), or a combination of the two. The cells were cultured in a humidified atmosphere (60\% relative humidity) containing $5 \% \mathrm{CO}_{2}$ at $37^{\circ} \mathrm{C}$. 
Lentiviral construction and generation of the active virus. DKK4 cDNA sequence was obtained from the National Center for Biotechnology Information (NCBI) database (http://www. ncbi.nlm.nih.gov/) and synthesized using the phosphoramidite method (11). The fragment was subsequently cloned into the Lv5 plasmid (Suzhou GenePharma Co., Ltd., Suzhou, China) to generate the expression construct. Expression construct and the Vira Power Lentiviral Packaging Mix (Thermo Fisher Scientific, Inc., Waltham, MA, USA) were co-transfected into the 293 FT cell line (Thermo Fisher Scientific, Inc.) to produce lentivirus. Lentiviral supernatants were subsequently harvested and used to infect colorectal cancer cell lines. Virus collection and propagation was performed according to the manufacturer's protocol.

Reverse transcription-quantitative polymerase chain reaction (RT-qPCR) and western blot analysis. Lentiviral DNA, free-from or containing DKK4, was transduced into 6 colorectal cancer cell lines. Cells were harvested and total RNA was extracted using the PureLink RNA Mini kit (Thermo Fisher Scientific, Inc.). RNA was reverse transcribed using the Super Script $^{\mathrm{TM}}$ III First-Strand Synthesis Super Mix for RT-qPCR (Thermo Fisher Scientific, Inc.). qPCR was performed using the SYBR ${ }^{\circledR}$ GreenER $^{\mathrm{TM}}$ qPCR Super Mix Universal (Thermo Fisher Scientific, Inc.), and DKK4 mRNA expression was normalized tothe internal control glyceraldehyde-3-phosphate dehydrogenase (GAPDH). The following primer sequences were used: GAPDH forward, 5'-ACAACTTTGGTATCGTGG AAGG-3' and reverse, 5'-GCCATCACGCCACAGTTTC-3'; DKK4 forward, 5'-ACGGACTGCAATACCAGAAAG-3' and reverse, 5'-CGTTCACACAGAGTGTCCCAG-3'. The data was normalized using the $2^{-\Delta \Delta \mathrm{Cq}}$ method (12).

The 12 colorectal cancer cell lines (6 overexpressing DKK4 and 6 controls) were washed with PBS (Invitrogen; Thermo Fisher Scientific, Inc.) and mixed with lysis buffer (Sigma-Aldrich; Merck Millipore). The mixtures were vortexed for $1 \mathrm{~min}$ and placed on ice for $30 \mathrm{~min}$. Following centrifugation $(10,000 \mathrm{x} g$ for $10 \mathrm{~min}, 4^{\circ} \mathrm{C}$ ), the dye-binding Bradford method (Bio-Rad Laboratories, Inc., Hercules, CA, USA) was used to quantify the protein. Equal amounts of protein ( $40 \mu \mathrm{g}$ protein/lane) were separated on a $10-12 \%$ sodium dodecyl sulfate (SDS) gel via polyacrylamide gel electrophoresis (PAGE) and transferred onto polyvinylidene difluoride (PVDF) membranes. The membranes were incubated with the primary anti-DKK4 rabbit monoclonal IgG antibody (ab172613; 1:1,000 dilution; Abcam, Cambridge, UK) and anti-GAPDH rabbit monoclonal $\mathrm{IgG}$ antibody (ab9483; 1:1,000 dilution; Abcam) separately, overnight at $4^{\circ} \mathrm{C}$. The secondary antibody was a horseradish peroxidase-labeled goat anti-rabbit IgG antibody (H+L) (A0208, 1:5,000 dilution; Beyotime Institute of Biotechnology, Haimen, China). The samples were incubated with the secondary antibody for $1 \mathrm{~h}$ at $37^{\circ} \mathrm{C}$. The signals were analyzed following treatment with 3,3', 5,5'-tetramethylbenzidine (TMB) substrate (P0211; Beyotime Institute of Biotechnology). The bands were visualized by the ChemiDocä Touch Imaging system (Bio-Rad Laboratories, Inc.).

Measurement of cell viability. Colorectal cancer cell lines were cultured in a 96-well microplate at a density of $5 \times 10^{3}$ cells/well for $24 \mathrm{~h}$ in a humidified atmosphere (60\% relative humidity) containing $5 \% \mathrm{CO}_{2}$ at $37^{\circ} \mathrm{C}$. The cells were subsequently divided into several groups and treated with 5-Fu, YN968D1 or both. The following drug concentrations were used: 0, 0.0128, 0.064, $0.32,1.6,8,40$ and $200 \mu \mathrm{g} / \mathrm{ml}$. Cell viability was assessed using the Cell Counting Kit- 8 assay at 3 days post-treatment according to the manufacturer's protocol. The absorbance at $450 \mathrm{~nm}$ was read using a 96-well plate reader in order to determine the cell viability.

Migration assay. Migration assays were performed in a 24-well Transwell ${ }^{\circledR}$ chamber (Corning Incorporated, Corning, NY, USA). A total of $40 \mu \mathrm{g} / \mathrm{ml} 5-\mathrm{Fu}$, YN968D1 or both were used and the Transwell chamber assays were performed according to the manufacturer's protocol.

Flow cytometric analysis of apoptotic cells. Fluorescence-activated cell sorting (FACS) was performed using the Annexin-V-fluorescein isothiocyanate (FITC) conjugate and binding buffer as standard reagents (Beyotime Institute of Biotechnology). The cells were exposed to the drugs $(20 \mu \mathrm{g} / \mathrm{ml}$ $5-\mathrm{Fu}$ and $40 \mu \mathrm{g} / \mathrm{ml}$ YN968D1) for $24 \mathrm{~h}$ and were subsequently collected for analysis. Flow cytometry was performed at $488 \mathrm{~nm}$ on a FACScanto flow cytometer (BD Biosciences, Franklin Lakes, NJ, USA). Fluorescent emission of FITC was measured at $515-545 \mathrm{~nm}$ and that of DNA-propidium iodide complexes at 564-606 nm. Cell debris was excluded from analysis by an appropriate forward light scatter threshold setting. Compensation was used wherever necessary.

Western blot analysis. Following drug treatment, cells were washed with PBS and mixed with lysis buffer. The mixtures were vortexed for $1 \mathrm{~min}$ and placed on ice for $30 \mathrm{~min}$. Following centrifugation $\left(10,000 \mathrm{x} g\right.$ for $\left.10 \mathrm{~min}, 4^{\circ} \mathrm{C}\right)$, the dye-binding Bradford method (Bio-Rad Laboratories, Inc.) was used to quantify the proteins. Equal amounts of protein ( $40 \mu \mathrm{g}$ protein/lane) were separated on a $10-12 \%$ SDS gel via PAGE and transferred onto PVDF membranes. The membranes were separately incubated with the primary anti-DKK4 rabbit monoclonal IgG antibody, anti-transcription factor AP-2 epsilon (TFAP2E) rabbit monoclonal IgG antibody (AV40023-100UG, 1:1,000 dilution; Sigma-Aldrich; Merck Millipore), anti-hypoxia-inducible factor- $2 \alpha$ (HIF2 $\alpha$ ) rabbit polyclonal IgG antibody (ab109616, 1:1,000 dilution; Abcam) and anti-GAPDH rabbit monoclonal IgG antibody (ab9483, 1:1,000 dilution; Abcam), overnight at $4^{\circ} \mathrm{C}$. The secondary antibody was a horseradish peroxidase-labeled goat anti-rabbit IgG antibody $(\mathrm{H}+\mathrm{L})$. The samples were incubated with the secondary antibody for $1 \mathrm{~h}$ at $37^{\circ} \mathrm{C}$. The signals were analyzed following treatment with TMB substrate and visualized by the ChemiDocä Touch Imaging system.

Statistical analysis. Differences between experimental groups were analyzed using the unpaired Student's t-test on Microsoft Office Excel 2010 (Microsoft Corporation, Redmond, WA, USA). $\mathrm{P}<0.05$ was considered to indicate a statistically significant difference.

\section{Results}

Overexpression of DKK4 in colorectal cancer cell lines. The DKK4 cDNA sequence was obtained from the NCBI database 
and the fragment synthesized using the chemical synthesis method. It was cloned into the Lv5 plasmid and co-transfected with packaging mix into 293FT cells to produce lentivirus. Lentiviral supernatant was harvested and used to infect 6 colorectal cancer cell lines, in order to induce DKK4 overexpression. RT-qPCR and western blot analyses were performed to measure the DKK4 expression in the various cell lines. The present RT-qPCR results demonstrated that DKK4 was upregulated in 4 cell lines: HCT116 (314.45-fold increase compared with the control; $\mathrm{P}=0.00027)$, HT29 (456.14-fold increase compared with the control; $\mathrm{P}=0.00052), \mathrm{Caco} 2$ (253.38-fold increase compared withthecontrol; $\mathrm{P}=0.00032$ ) and Colo205 (204.89-fold increase compared with the control; $\mathrm{P}=0.00001$ ) (Fig. 1A). Similar results were observed following western blot analysis (Fig. 1B). The mRNA and protein expression levels observed indicated that DKK4 was being overexpressed in those 4 cell lines. Therefore subsequent research made use of only those 4 cell lines to investigate the role of DKK4 in the development of resistance to chemotherapy in colorectal cancer cells.

Effect of DKK4 on cell viability. As 5-Fu is one of the most widely used drugs in colorectal cancer treatment and YN968D1 is a tyrosine kinase inhibitor used in metastatic cancer, they were selected to investigate the role of DKK4 in resistance to chemotherapy.

Cell lines were treated with varying doses of 5-Fu, YN968D1 or both to assess cell viability. The present results demonstrated that in the 4 selected cell lines, cell viability decreased in a dose-dependent manner in the control and DKK4-overexpressing cells (Fig. 2). Furthermore, the half maximal inhibitory concentration $\left(\mathrm{IC}_{50}\right)$ data for the HCT116 and HT29 cell lines was significantly increased $(\mathrm{P}=0.00032$ for combined drug treatment inHCT116 cells; $\mathrm{P}=0.01426$ for 5-Fu treatment in HT29 cells) in the DKK4 over-expressing cells compared with the control cells following drug treatment, which suggest that DKK4 enhances colorectal cancer cell resistance tochemotherapy (Fig. 2). The present results demonstrated that treatment with 5-Fu, YN968D1 or both decreases the viability of cancer cells, however DKK4 over-expressing cells demonstrated higher drug resistance.

Effect of DKK4 on cell migration. A Transwell chamber assay was performed to assess the effect of DKK4 expression on cell migration in colorectal cancer cell lines. The results are presented in Table I. The migration percentage in HCT116 cells of the DKK4 over-expressing cells was $88.7 \pm 1.5,81.0 \pm 1.0$ and $71.7 \pm 2.1$ following treatment with $5-\mathrm{Fu}, \mathrm{YN} 968 \mathrm{D} 1$ or both, respectively, while the control migration percentages were $61.7 \pm 1.5,57.3 \pm 1.5$ and $32.0 \pm 1.0$, respectively. However, in the HT29 cell line the migration percentages in the DKK4 over-expressing cells were reduced compared with the control. In the remaining cell lines, the differences in migration percentages between DKK4 over-expressing cells and control cells were negligible. The present data suggests that DKK4 may have an effect on cell migration in cancer cells following drug treatment.

Effect of DKK4 on cell death. The aforementioned data demonstrated that DKK4 had an effect on cell viability and migration, therefore the effect of DKK4 expression on cell
A

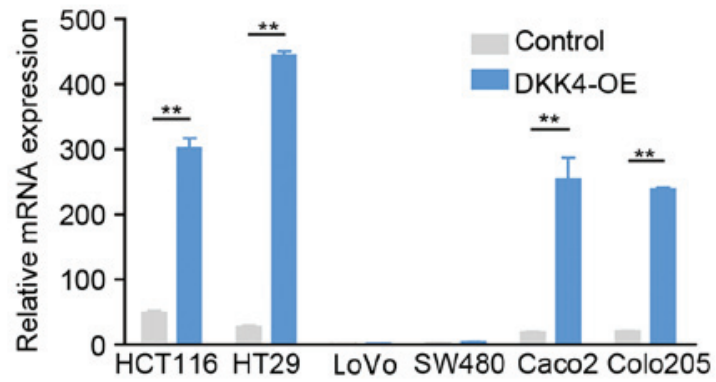

\section{B HCT116 HT29 LoVo SW480 Caco2 Colo205 $\overline{C t r l} \mathrm{OE} \overline{\mathrm{Ctrl} O E} \overline{\mathrm{Ctrl} O \mathrm{OE}} \overline{\mathrm{Ctrl} \mathrm{OE}} \overline{\mathrm{Ctrl} \mathrm{OE}} \overline{\mathrm{Ctrl} O E}$ DKK4}

GAPDH

Figure 1. Overexpression of DKK4 in 6 colorectal cancer cell lines (A) Reverse transcription-quantitative polymerase chain reaction analysis of DKK4 mRNA relative expression and (B) western blot analysis of DKK4 protein levels in cell lines following treatment with 5-Fu, YN968D1, both or neither. GAPDH was used as the internal control. DKK4, dickkopf-related protein 4; OE, overexpressing; Ctrl, control; GAPDH, glyceraldehyde 3 -phosphate dehydrogenase. Data is presented as the mean \pm standard error. ${ }^{* *} \mathrm{P}<0.005$.

death was subsequently analyzed. Due to data obtained from the cell viability and migration assays, the HCT116 and HT29 cell lines were selected for subsequent study. FACS analysis was used to evaluate the apoptotic status of cells (Fig. 3). The percentage of apoptotic cells in the HCT116 cell line following $5-\mathrm{Fu}$ treatment was $44.32 \%$ in the DKK4-overexpressing cells and $50.08 \%$ in the control cells $(\mathrm{P}=0.0011)$. Following YN968D1 treatment the percentage of apoptotic cells was $19.49 \%$ in the DKK4 over-expressing cells and $36.38 \%$ in the control cells $(\mathrm{P}=0.0052)$. Following combined treatment with the two drugs, the percentage of apoptotic cells was $53.83 \%$ in the DKK4 over-expressing cells and $70.74 \%$ in the control cells. The results indicated that the HCT116 DKK4 over-expressing cells demonstrated increased resistance to drugs compared with the control cells $(\mathrm{P}=0.0006$; Fig. 3A). The percentage of apoptotic cells in the HT29 cell line following 5-Fu treatment was $25.91 \%$ in the DKK4 over-expressing cells and $50.42 \%$ in the control cells $(\mathrm{P}=0.0027)$. Following YN968D1 treatment the percentage of apoptotic cells was $15.7 \%$ in the DKK4 over-expressing cells and $44.09 \%$ in the control cells $(\mathrm{P}=0.0094)$. Following combined treatment with the two drugs, the percentage of apoptotic cells was $50.77 \%$ in the DKK4 over-expressing cells and $70.09 \%$ in the control cells $(\mathrm{P}=0.0012)$. The results were almost identical in the HT29 cell line, except there was less cell death in the HT29 DKK4 over-expressing cells following drug treatment (Fig. 3B). The present data indicate that DKK4 may decrease the number of apoptotic colorectal cancer cells following treatment with chemotherapy drugs.

Effect of DKK4 on other cancer-associated genes. TFAP2E is a member of the AP-2 transcription factor family and 
Table I. Effect of DKK4 on cell migration.

Migration (\%)

\begin{tabular}{|c|c|c|c|c|c|c|c|}
\hline \multicolumn{2}{|c|}{ HCT116 } & \multicolumn{2}{|c|}{ HT29 } & \multicolumn{2}{|c|}{ Caco2 } & \multicolumn{2}{|c|}{ Colo205 } \\
\hline Control & DKK4-OE & Control & DKK4-OE & Control & DKK4-OE & Control & DKK4-OE \\
\hline $61.7 \pm 1.5$ & $88.7 \pm 1.5$ & $72.3 \pm 2.1$ & $54.0 \pm 2.6$ & $79.0 \pm 3.0$ & $135.0 \pm 8.9$ & $109.7 \pm 3.8$ & $105.3 \pm 1.7$ \\
\hline $57.3 \pm 1.5$ & $81.0 \pm 1.0$ & $88.7 \pm 0.6$ & $78.3 \pm 3.2$ & $206.7 \pm 7.6$ & $130.3 \pm 9.0$ & $109.3 \pm 0.6$ & $100.7 \pm 0.6$ \\
\hline $32.0 \pm 1.0$ & $71.7 \pm 2.1$ & $63.7 \pm 1.5$ & $39.6 \pm 2.9$ & $132.7 \pm 7.5$ & $67.0 \pm 4.4$ & $104.8 \pm 1.9$ & $104.7 \pm 3.9$ \\
\hline
\end{tabular}

Data are presented as the mean \pm standard error. DKK4, dickkopf-related protein 4; 5-Fu, 5-fluorouracil; YN-1, YN968D1; OE, overexpressing.

A

DKK4-OE

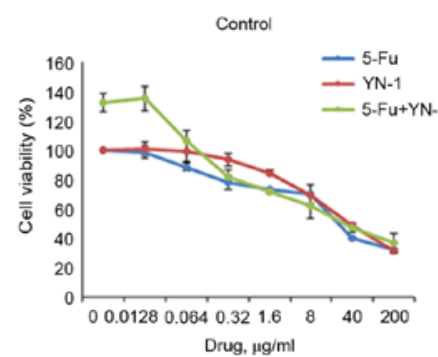

B

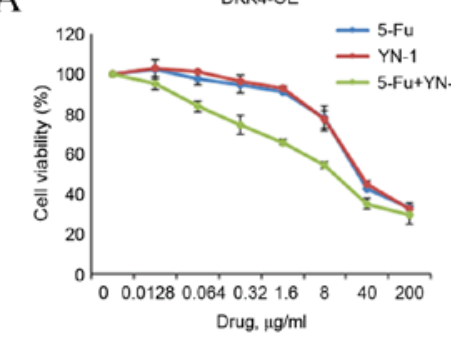

$\mathrm{C}$
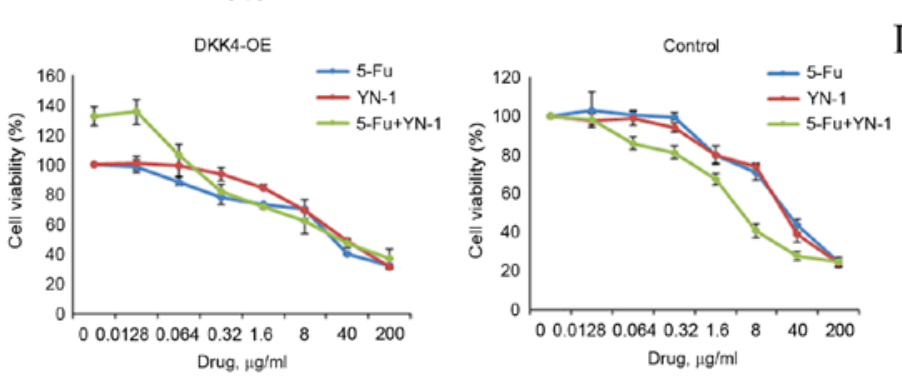

\begin{tabular}{|c|c|c|}
\hline IC $_{50}(\mathrm{\mu g} / \mathrm{ml})$ & DKK4 & LV-NC \\
\hline $5-F u$ & $15.52 \pm 0.74$ & $12.93 \pm 0.26$ \\
\hline $\mathrm{YN}-1$ & $14.99 \pm 5.02$ & $10.87 \pm 0.91$ \\
\hline $5-\mathrm{Fu}+\mathrm{YN}-1$ & $3.59 \pm 0.85^{*}$ & $0.20 \pm 0.03$ \\
\hline
\end{tabular}

E
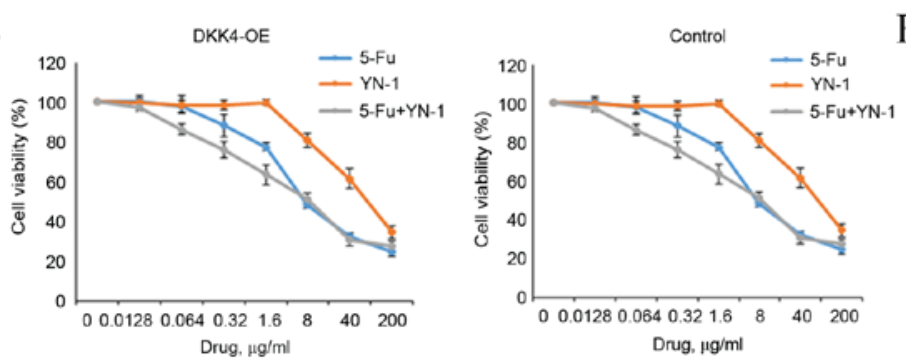

$\mathrm{F}$

\begin{tabular}{|c|c|c|}
\hline $\mathrm{IC}_{5_{0}}(\mu \mathrm{g} / \mathrm{ml})$ & DKK4 & LV-NC \\
\hline $5-\mathrm{Fu}$ & $32.57 \pm 2.34^{*}$ & $11.57 \pm 1.72$ \\
\hline $\mathrm{YN}-1$ & $39.27 \pm 7.69$ & $15.87 \pm 7.66$ \\
\hline $5-\mathrm{Fu}+\mathrm{YN}-1$ & $9.17 \pm 5.47$ & $2.54 \pm 0.32$ \\
\hline
\end{tabular}

G
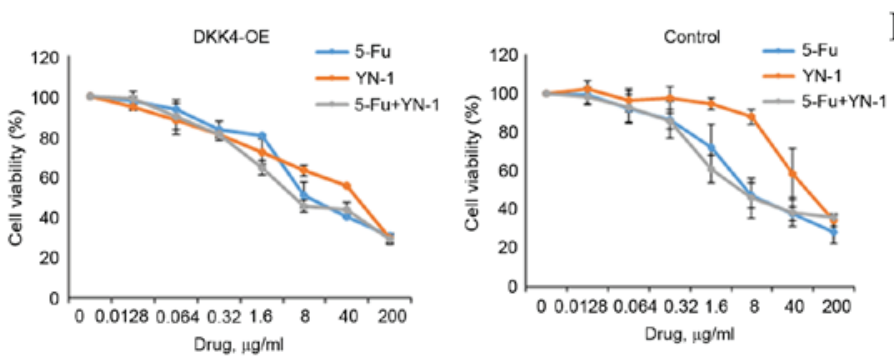

$\mathrm{H}$

\begin{tabular}{|c|c|c|}
\hline $\mathrm{IC}_{\text {so }}(\mu \mathrm{g} / \mathrm{ml})$ & DKK4 & LV-NC \\
\hline $5-\mathrm{Fu}$ & $3.84 \pm 0.77$ & $2.67 \pm 0.32$ \\
\hline $\mathrm{YN}-1$ & $34.05 \pm 2.35$ & $34.94 \pm 8.37$ \\
\hline $5-\mathrm{Fu}+\mathrm{YN}-1$ & $2.50 \pm 1.52$ & $1.16 \pm 0.17$ \\
\hline
\end{tabular}

Figure 2. Effect of treatment with 5-Fu, YN-1 or both on cell viability, and the $\mathrm{IC}_{50}$ value in DKK4-overexpressing and control cells. (A) Cell viability and (B) $\mathrm{IC}_{50}$ in the HCT116 cell line; (C) cell viability and (D) $\mathrm{IC}_{50}$ in the HT29 cell line; (E) cell viability and (F) IC ${ }_{50}$ in the Caco2 cell line; and (G) cell viability and $(\mathrm{H}) \mathrm{IC}_{50}$ in the Colo205 cell line. Fu-5, 5-fluorouracil; YN-1, YN968D1; $\mathrm{IC}_{50}$ half maximal inhibitory concentration; DKK4, dickkopf-related protein 4. LV-NC, lentivirus negative control. Data is presented as the mean \pm standard error. ${ }^{*} \mathrm{P}<0.05$ vs. the control.

has an important role in cancer biology. A previous report demonstrated that TFAP2E hypermethylation is associated with resistance to chemotherapy in colorectal cancer and that TFAP2E-dependent resistance to chemotherapy is mediated by DKK4 (9). In order to determine whether the expression pattern of this gene was altered, western blotting 

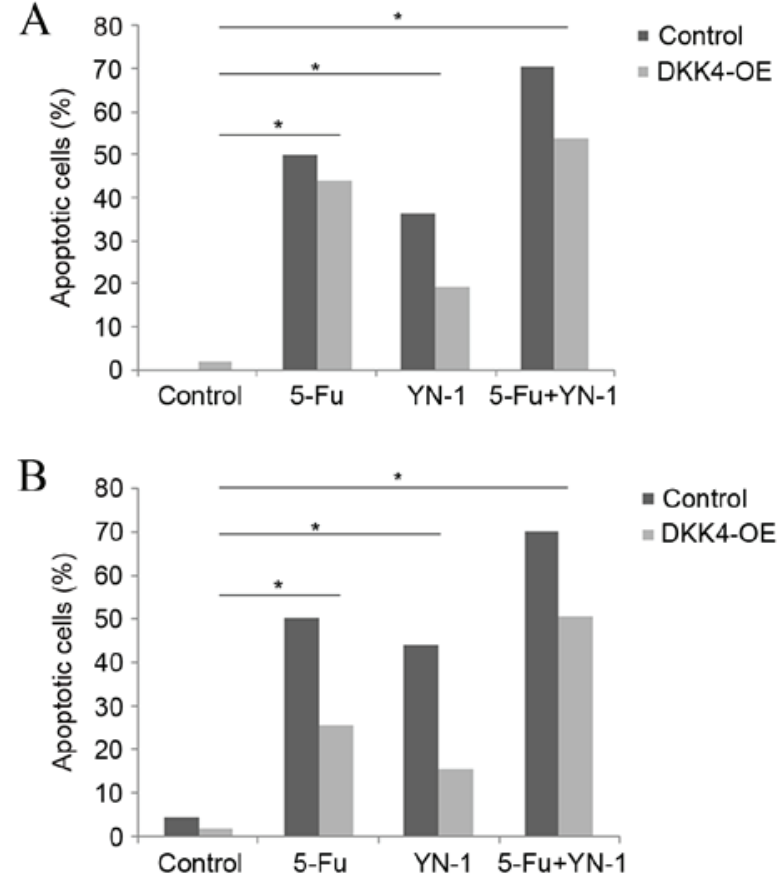

Figure 3. Effect of treatment with 5-Fu, YN-1 or both on cell death in DKK4-overexpressing and control cells. Apoptosis was analyzed using fluorescence-activated cell sorting. The percentage of apoptotic cells in (A) HCT116 cells and (B) HT29 cells. Data is presented as the mean \pm standard error. ${ }^{*} \mathrm{P}<0.05$. 5-Fu, 5-fluorouracil; YN-1, YN968D1; DKK4, dickkopf-related protein 4; OE, overexpressing.

was performed in the present study. Compared with the control, the TFAP2E expression level was increased in the DKK4 over-expressing cells of both untreated cell lines when compared with the control cells (Fig. 4). Following any form of drug treatment, TFA2E expression was increased in both cell lines compared to the untreated cell lines. This increase was more marked in the DKK4 over-expressing cells compared with the control cells (Fig. 4).

Another gene investigated in the present study was HIF $2 \alpha$. HIF2 $\alpha$ belongs to the basic helix-loop-helix/Per-ARNT-Sim domain transcription factor family and has clinical value in predicting cancers $(13,14)$. For example, Koukourakis et al (14) observed that HIF proteins may have value in predicting early esophageal cancer and the clinical responses to photodynamic therapy (PDT). It was reported that the HIF proteins were expressed in the normal epithelium around the early-stage esophageal tumor, but not in the normal esophagus (14). Furthermore, it was revealed that following treatment with PDT and radiotherapy, the expression levels of HIF proteins were highly upregulated, which indicated that the HIF proteins may enhance the resistance of tumors to PDT (14). The present results demonstrated that drug treatment had a negligible effect on HIF2 $\alpha$ expression in the HCT116 cell line compared with untreated HCT116 cells (Fig. 4A). Drug treatment upregulated HIF2 $\alpha$ expression in the HT29 cell line compared with the untreated HT29 cells (Fig. 4B).

The present results indicate that the expression patterns of certain cancer-associated genes is altered between DKK4 over-expressing cells and control cells, but whether this alteration is dependent on DKK4 remains to be elucidated.
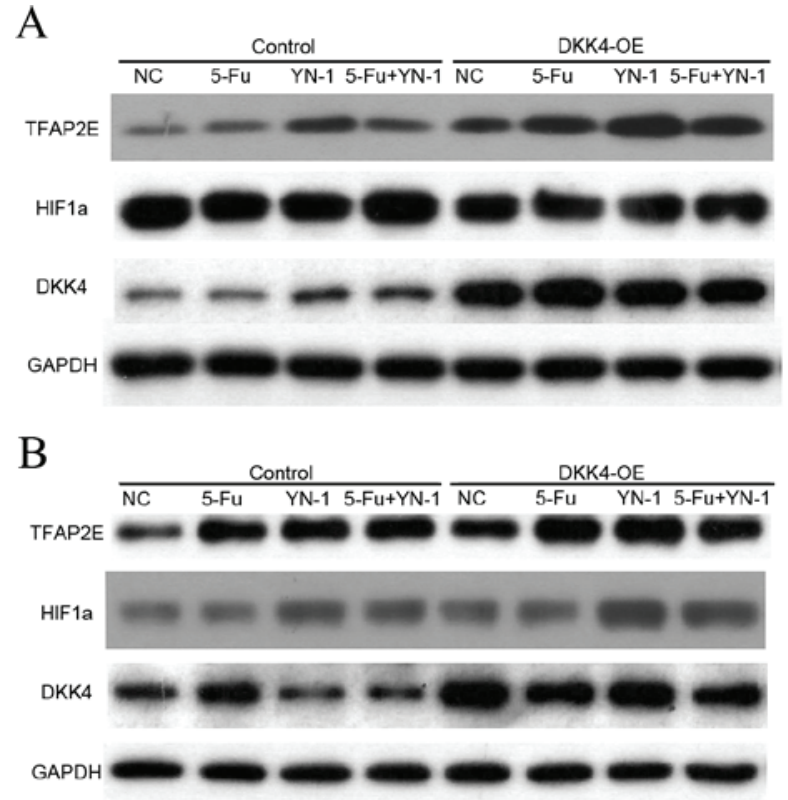

Figure 4. Western blot analysis of DKK4, TFAP2E and HIF2a in DKK4-overexpressing and control cells following drugtreatment. Proteins were detected by western blot in (A) HCT116 cell lines and (B) HT29 cell lines following treatment with $5-\mathrm{Fu}, \mathrm{YN}-1$ or both. GAPDH was used as the internal control. DKK4, dickkopf-related protein 4; TFAP2E, transcription factor AP-2 epsilon; HIF2a, hypoxia-inducible factor-2alpha; GAPDH, glyceraldehyde 3-phosphate dehydrogenase; NC, negative control; OE, overexpressing; 5-Fu, 5-fluorouracil; YN-1, YN968D1.

\section{Discussion}

Colorectal cancer has one of the highest mortality rates and is often not diagnosed until it has reached an advanced stage (1). Chemotherapy is commonly utilized in cancer treatment and 5 -Fu is widely used (3). The drug 5-Fu induces apoptosis in cancer and normal cells (3). YN968D1 is a tyrosine kinase inhibitor that is thought to inhibit angiogenesis in cancer cells (6).

Previous studies have shown that in human colon cancer, DKK4 has a role in cancer development. The expression of DKK4 is induced in colorectal cancer, and this promotes tumor cells invasion and angiogenesis (15). Baehs et al (10) reported that DKK4 was able to inhibit the growth of colorectal cancer cells and cell cycle progression. In chemoresistance research, studies have revealed that overexpression of DKK4 enhances resistance to 5-Fu, and this resistance may function in TFAP2E-dependent resistance in colorectal cancer cells (9). The present study aimed to investigate the role of DKK4 in cancer chemoresistance not only to 5-Fu, but also to another drug or a combination of the 2 . In the present study, DKK4 was over expressed in several colorectal cancer cell lines and its expression level was analyzed by RT-qPCR and western blotting. Of the original 6, 4 cell lines exhibited high expression of DKK4.

Cell proliferation assays demonstrated that cell viability decreased in a dose-dependent manner following drug treatment and that the $\mathrm{IC}_{50}$ of drugs was increased in DKK4 over-expressing cells compared with control cells in both the HCT116 and HT29 cell lines. The present results suggest that DKK4 enhances the resistance of tumor cells to chemotherapy 
drugs. The results of the present study are similar to those of Ebert et al (9), who observed that overexpression of DKK4 was able to enhance resistance to 5-Fu. Analysis of Transwell chamber assays indicated that DKK4 had an effect on the migration of colorectal cancer cells following treatment with chemotherapy drugs. Furthermore, FACS demonstrated that DKK4 decreased the percentage of apoptotic cells following treatment with chemotherapy drugs. The present results suggest that DKK4 may enhance resistance to chemotherapy in colorectal cancer cells. In addition to this, western blotting was performed to analyze TFAP2E and HIF2 $\alpha$ expression in DKK4-overexpressing cells and control cells following drug treatment. The present results indicated that treatment with a single drug/drug combination alters the expression pattern of TFAP2E and HIF2 $\alpha$; however, whether this alteration is dependent on DKK4 remains to be elucidated.

In conclusion, the present study indicates that DKK4 may have a role in the development of resistance to chemotherapy drugs, including 5-Fu and YN968D1, in colorectal cancer cells.

\section{Acknowledgements}

This study was supported by the Shangshai Minhang District Health and Family Planning Commission (Shanghai, China; grant no. 2015MW22) and the Program for Shanghai Minhang District Outstanding Academic Leader (Shanghai, China; grant no. 201511).

\section{References}

1. Tsukihara H, Nakagawa F, Sakamoto K, Ishida K, Tanaka N, Okabe H, Uchida J, Matsuo K and Takechi T: Efficacy of combination chemotherapy using a novel oral chemotherapeutic agent, TAS-102, together with bevacizumab, cetuximab, or panitumumab on human colorectal cancer xenografts. Oncol Rep 33: 2135-2142, 2015

2. Wang $H$, Zhang L, Yang X, Jin Y, Pei S, Zhang D, Zhang H, Zhou B, Zhang Y and Lin D: PUMA mediates the combinational therapy of 5-FU and NVP-BEZ235 in colon cancer. Oncotarget 6: 14385-14398, 2015.

3. Longley DB, Harkin DP and Johnston PG: 5-fluorouracil: Mechanisms of action and clinical strategies. Nat Rev Cancer 3 : 330-338, 2003
4. Zhang N, Yin Y, Xu SJ and Chen WS: 5-Fluorouracil: Mechanisms of resistance and reversal strategies. Molecules 13: 1551-1569, 2008.

5. Xi Y, Formentini A, Nakajima G, Kornmann $\mathrm{M}$ and $\mathrm{Ju} \mathrm{J}$ : Validation of biomarkers associated with 5-fluorouracil and thymidylate synthase in colorectal cancer. Oncol Rep 19: 257-262, 2008.

6. Tian S, Quan H, Xie C, Guo H, Lü F, Xu Y, Li J and Lou L: YN968D1 is a novel and selective inhibitor of vascular endothelial growth factor receptor-2 tyrosine kinase with potent activity in vitro and in vivo. Cancer Sci 102: 1374-1380, 2011.

7. Li J, Zhao X, Chen L, Guo H, Lv F, Jia K, Yv K, Wang F, Li C, Qian J, et al: Safety and pharmacokinetics of novel selective vascular endothelial growth factor receptor-2 inhibitor YN968D1 in patients with advanced malignancies. BMC Cancer 10: 529, 2010.

8. Maehata T, Taniguchi H, Yamamoto H, Nosho K, Adachi Y, Miyamoto N, Miyamoto C, Akutsu N, Yamaoka S and Itoh F: Transcriptional silencing of Dickkopf gene family by $\mathrm{CpG}$ island hypermethylation in human gastrointestinal cancer. World J Gastroenterol 14: 2702-2714, 2008.

9. Ebert MP, Tänzer M, Balluff B, Burgermeister E, Kretzschmar AK, Hughes DJ, Tetzner R, Lofton-Day C, Rosenberg R, Reinacher-Schick AC, et al: TFAP2E-DKK4 and chemoresistance in colorectal cancer. N Engl J Med 366: 44-53, 2012.

10. Baehs S, Herbst A, Thieme SE, Perschl C, Behrens A, Scheel S, Jung A, Brabletz T, Göke B, Blum H and Kolligs FT: Dickkopf-4 is frequently down-regulated and inhibits growth of colorectal cancer cells. Cancer Lett 276: 152-159, 2009.

11. Mandecki W, Mollison KW, Bolling TJ, Powell BS, Carter GW and Fox JL: Chemical synthesis of a gene encoding the human complement fragment C5a and its expression in Escherichia coli. Proc Natl Acad Sci USA 82: 3543-3547, 1985.

12. Livak KJ and Schmittgen TD: Analysis of relative gene expression data using real-time quantitative PCR and the 2(-Delta Delta C(T)) method. Methods 25: 402-408, 2001.

13. Swartz JE, Pothen AJ, Stegeman I, Willems SM and Grolman W: Clinical implications of hypoxia biomarker expression in head and neck squamous cell carcinoma: A systematic review. Cancer Med 4: 1101-1016, 2015

14. Koukourakis MI, Giatromanolaki A, Skarlatos J, Corti L, Blandamura S, Piazza M, Gatter KC and Harris AL: Hypoxia inducible factor (HIF-1a and HIF-2a) expression in early esophageal cancer and response to photodynamic therapy and radiotherapy. Cancer Res 61: 1830-1832, 2001.

15. Pendás-Franco N, García JM, Peña C, Valle N, Pálmer HG, Heinäniemi M, Carlberg C, Jiménez B, Bonilla F, Muñoz A and González-Sancho JM: DICKKOPF-4 is induced by $\mathrm{TCF} /$ beta-catenin and upregulated in human colon cancer, promotes tumour cell invasion and angiogenesis and is repressed by lalpha, 25-dihydroxyvitamin D3. Oncogene 27: 4467-4477, 2008. 